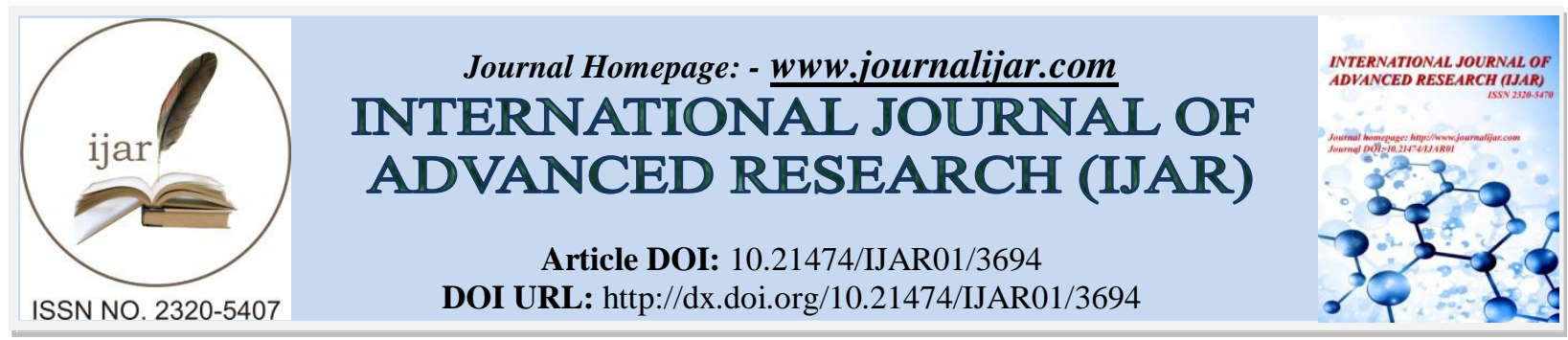

RESEARCH ARTICLE

\title{
COMPRESSION DU NERF ULNAIRE AU POIGNET PAR UNE TUMEUR A CELLULES GEANTES : A PROPOS D'UN CAS ULNAR NERVE COMPRESSION IN GUYON'S CANAL BY GIANT CELL TUMOR: REPORT A CASE.
}

\author{
Omar Margad ${ }^{1}$ and Nabile Bousselmame 2 . \\ 1. Service de traumatologie orthopédie de l'hôpital militaire Avicenne Marrakech. \\ 2. Cabinet médical, Appt.12, Imm.23, avenue bin al ouidane, Agdal, Rabat.
}

\section{Manuscript Info}

Manuscript History

Received: 13 January 2017

Final Accepted: 09 February 2017

Published: March 2017

Key words:-

Guyon's canal, giant cell tumor.

\section{Abstract}

A case of ulnar nerve compression in Guyon's canal is reported in 43year-old woman. Magnetic resonance imaging demonstrated a giant cell tumor confirmed by histological examination. Surgical treatment had fully restored sensory and partial motor function of the right hand.

\section{Introduction:-}

La compression du nerf ulnaire n'est pas rare, c'est la $2^{\circ}$ neuropathie compressive après celle du nerf médian au niveau du canal carpien. Si le site de compression le plus fréquent est le coude, la compression au niveau du canal de Guyon n'est pas exceptionnelle et souvent et souvent due à une cause locale. Nous rapportons le cas d'une patiente présentant un syndrome du canal de Guyon révélant une tumeur à cellules géantes.

\section{Observation:-}

Il s'agit d'une patiente âgée de 43ans, droitière, femme au foyer, ayant consulté pour des paresthésies avec fourmillements et picotements au niveau du territoire du nerf ulnaire. L'examen clinique a révélé une atrophie de l'éminence hypothénarienne, un signe de wartenberg positif [figure 1], une diminution de la sensibilité des deux derniers doigts et une absence de l'abduction et de l'adduction des deux derniers doigts. La radiographie standard a objectivé la masse en regard de l'éminence hypothénarienne et l'IRM a précisé son origine au niveau de la gaine des fléchisseurs comprimant et refoulant le pédicule ulnaire [figure 2]. L'exploration chirurgicale a montré une tuméfaction jaunâtre, encapsulée, se développant à partir de la gaine des fléchisseurs des quatres derniers doigts [figure 3]. L'intervention a consisté en une résection de la totalité de tumeur et la neurolyse du nerf ulnaire jusqu'à sa division dans le hiatus pisi-unciformien puis section du ligament pisi-unciformien et de l'arcade fibreuse des hypothénariens. Les suites postopératoires ont été simples, la patiente a démarré la rééducation fonctionnelle une semaine après l'intervention. L'examen anatomopathologique a confirmé le diagnostic de tumeur à cellules géantes. Un an après son intervention la patiente a récupéré complètement la sensibilité de la main et partiellement la force motrice. 


\section{Discussion:-}

La compression du nerf ulnaire dans le canal de Guyon n'est pas exceptionnelle. Décrite pour la première fois par l'urologiste français Félix Guyon en 1861 [1]. Ses limites anatomiques bien précises conditionnant la symptomatologie clinique ont permis de diviser le canal de Guyon en trois zones [2,3].

La zone I, correspond à la zone ou le nerf ulnaire va se diviser en ses deux branches terminales, suerficielle sensitive et profonde motrice. La compression en cette zone va produire soit un déficit sensitivo-moteur, soit sensitif ou moteur.

La zone II, se situe autour de la branche motrice.la compression à ce niveau sera responsable d'une symptomatologie purement motrice.

La zone III, autour de la branche superficielle. Sa symptomatologie sera purement sensitive.

Plusieurs étiologies peuvent être responsables du syndrome du canal de Guyon [3]. Les tumeurs à cellules géantes viennent au $2^{\circ}$ rang après les kystes synoviaux. Ces tumeurs bénignes malgré leur grande fréquence au niveau de la main [4] sont mal diagnostiquées en préopératoire. Dans une revue de la littérature seulement quelques cas de tumeurs à cellules géantes responsable de syndrome du canal de Guyon ont été décrits [5-11]. Ces tumeurs bénignes se développent à partir de la gaine synoviale des tendons et des articulations du poignet et de la main, et plus précisément au niveau du dédoublement externe de la gaine ce qui les différencie des synovites villonodulaires à développement interne [12]. Les tumeurs à cellules géantes peuvent être localisées ou diffuses pouvant intéresser également le genou, la hanche...

Pour le diagnostic, l'électromyogramme (EMG) reste le maitre examen complémentaire des syndromes compressifs. Dans notre cas, il a objectivé un allongement de la conduction sensitive du nerf ulnaire à sa partie distale et une atteinte de la branche profonde motrice. L'IRM a un rôle prépondérant dans le diagnostic étiologique, elle permet de faire le diagnostic différentiel avec le sarcome des parties molles qui produit un signal d'intensité égale ou supérieure à celle de la graisse en T2, alors que les tumeurs à cellules géantes ont un signal entre le muscle et la graisse en T2 [13]. De plus, les tumeurs à cellules géantes se rehaussent après injection de Gadolinium du à la prolifération des capillaires dans le stroma, ce qui les différencient des kystes synoviaux [14]. Le traitement des tumeurs à cellules géantes est essentiellement chirurgical et repose sur l'excision complète de la tumeur avec un risque de récidive moyen de 26,5\% durant les deux premières années [15]. Ces récidives sont favorisées par l'excision incomplète, la localisation au niveau du pouce ou l'érosion de l'os [16]. L'irradiation, autrefois utilisé pour éliminer le risque des récidives est actuellement dépassée vu ses effets secondaires, en particulier la contracture de la main [17]. A coté de l'exérèse de la tumeur, on procède à une neurolyse du nerf jusqu'à sa division dans le hiatus pisi-unciformien où la section de l'arcade fibreuse des hypothénariens et du ligament pisi-unciformien est indispensable [18].

\section{Conclusion:-}

Le syndrome du canal de Guyon est rare, souvent due à une cause locale. Les tumeurs à cellules géantes fréquentes au niveau de la main doivent faire partie des diagnostics différentiels dominés par les kystes synoviaux. L'IRM est très utile dans l'orientation étiologique. 


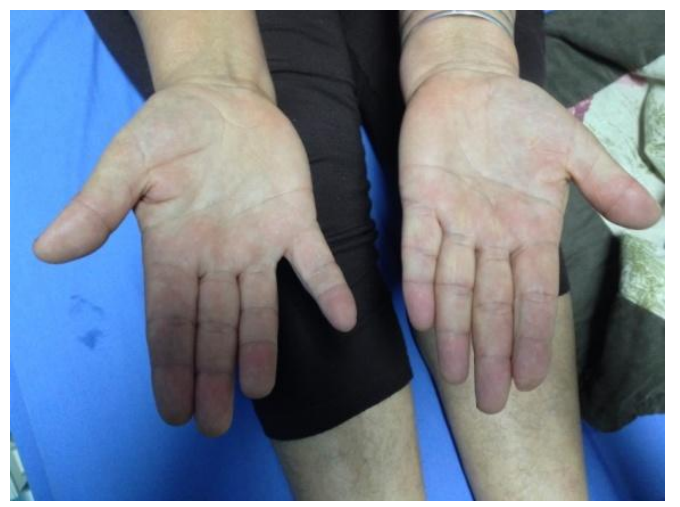

Figure 1:- Atrophie de l'éminence hypothénarienne et signe de Wartenberg.
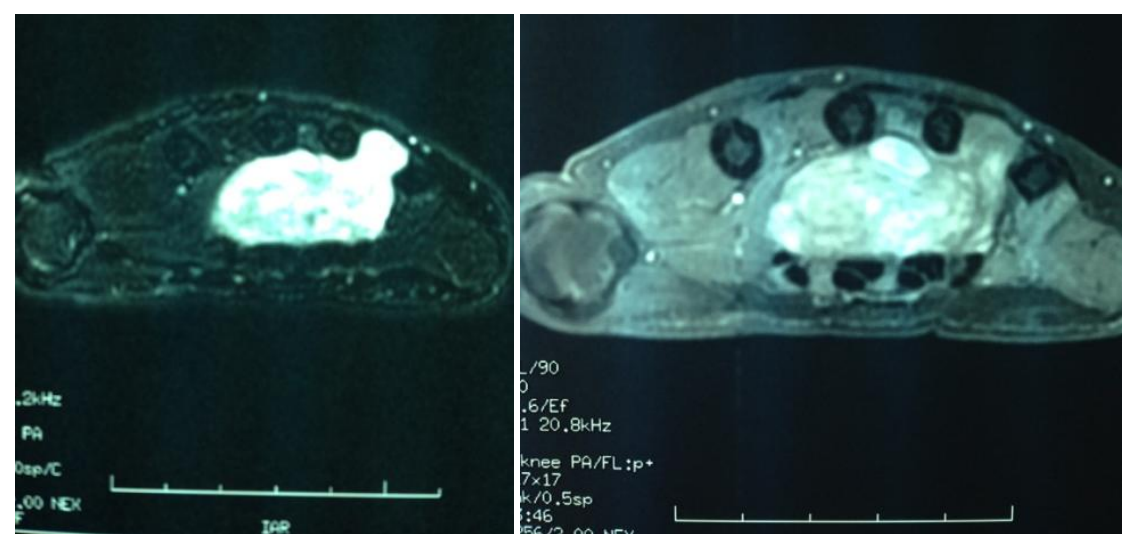

Figure 2:- IRM de la main objectivant la tumeur et son retentissement sur les structures avoisinantes.

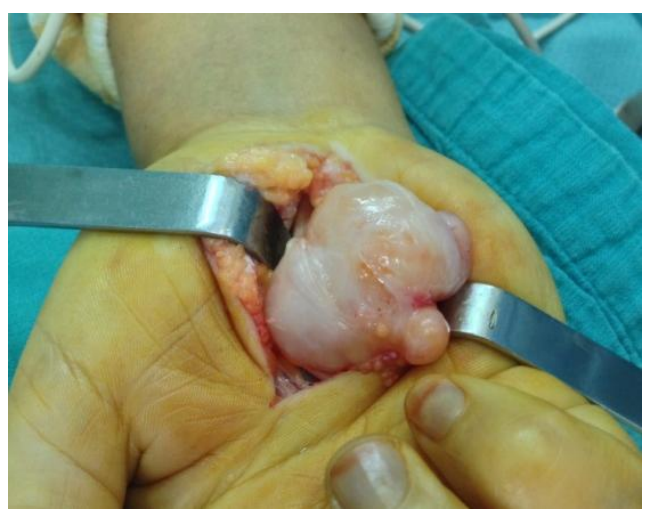

Figure 3:- Excision de la tumeur.

\section{References:-}

1. Guyon. : Note sur une disposition anatomique propre à la face antérieure de la région du poignet et non décrite par le docteur. Bull. Soc. Anat.de Paris, 6:184-186, 1861.

2. Gross MS, Gelberman RH. The anatomy of the distal ulnar tunnel. Clin Orthop Relat Res. 1985; (196):238-47.

3. Shea JD, McClain EJ. Ulnar-nerve compression syndromes at below the wrist. J Bone Joint Surg Am. 1969; 51(6):1095-103.

4. Karasick D, Karasick S. Giant cell tumor of tendon sheat: spectrum of radiologic findings. Skeletal Radiol. 1992; 21(4): 219-24.

5. Bundy PG, Regan PJ, Roberts AH. Localized nodular synovitis: a rare cause of ulnar nerve compression in Guyon's canal. J Hand Surg [AM] 1992; 17(4): 663-64.

6. Hayes CW, Jr. ulnar tunnel syndrome from giant cell tumor of tendon sheat: a case report. J Hand Surg [AM] 1978; 3(2): 187-88. 
7. Milberg P, Kleinert HE. Giant cell tumor compression of the deep branch of the ulnar nerve. Ann Plast Surg. 1980; 4(5): 426-29.

8. Nucci F, Artico M, Antonini G, Millefiorini M, Bastianello S, Bozzao L. Compression of the ulnar nerve in Guyon's canal by a giant cell tumor. Zentralbl neurochir.1989; 50(3-4): 196-98.

9. Rafecas JC, Daube JR, Ehman RL. Deep branch ulnar neuropathy due to giant cell tumor: report of case. Neurology. 1988; 38(2): 327-29.

10. Rengachary SS, Arjunan K. Compression of the ulnar nerve in Guyon's canal by a soft tissue giant cell tumor.Neurosergery.

11. Ben S.Francisco, BA and Jayant P.Agarwal, MD. Eplasty.2009; 9: e8.

12. Jaffe HL, Lichtenstein L, Sutro CJ. Pigmented villonodular synovitis, bursitis and tenosynovitis: A discussion of the synovial and bursal equivalents of the tenosynovial lesion commonly denoted as xanthoma, xanthogranuloma, giant cell tumor or myeloplaxoma of the tendon sheath, with some consideration of this tendon sheath lesion itself. Arch Pathol. 1941; 31: 731-65.

13. Kitagawa $\mathrm{Y}$, Ito $\mathrm{H}$, Amano $\mathrm{Y}$, Sawaizumi T, Takeuchi T. MR imaging for preoperative diagnosis and assessment of local tumor extent on localized giant cell tumor of tendon sheath. Skeletal Radiol. 2003; 32(11): 633-38.

14. Khan S, Neumann C, Steinbach L, Harrington K. MRI of giant cell tumor of tendon sheath of the hand: a report of three cases. Eur Radiol. 1995; 5(4): 467-70.

15. Reilly KE, Stern PJ, Dale JA. Recurrent giant cell tumors of the tendon sheath. J Hand Surg [AM] 1999; 24(6): 1298-302.

16. Grover R, Grobbelaar AO, Richman PI, Smith PJ. Measurement of invasive potential provides an accurate prognostic marker for giant cell tumor of tendon sheath Hand Surg [BR] 1998; 23(6): 728-31.

17. Walsh EF, Mechrefe A, Akelman E, Schiller AL. Giant cell tumor of tendon sheath. Am J Orthop. 2005; 34(3): $116-21$. 